\title{
COMMUNICATION
}

\section{Rejuveinix Mitigates Sepsis-Associated Oxidative Stress in the Brain of Mice: Clinical Impact Potential in COVID-19 and Nervous System Disorders}

Fatih M. Uckun ${ }^{1,2^{*}}$, Mehmet Tuzcu ${ }^{3}$, Marcus Gitterle ${ }^{4}$, Michael Volk $^{1}$, Kazim Sahin $^{5}$

${ }^{1}$ Drug Discovery Program, Reven Pharmaceuticals, Westminster, CO 80234, USA; fatih.uckun@reven.com (F.M.U); michael.volk@reven.com (M.V)

${ }^{2}$ Department of Developmental Therapeutics, Immunology, and Integrative Medicine, Ares Pharmaceuticals, St. Paul, MN 55110, USA;

${ }^{3}$ Department of Biology, Faculty of Science, Firat University, Elazig 23119, Turkey; mtuzcu@firat.edu.tr (M.T)

${ }^{4}$ Department of Surgery, Christus Health System, Santa Rosa Hospital, New Braunfels, TX 78130, USA; marcus.gitterle@christushealth.org

${ }^{5}$ Department of, Faculty of Veterinary, Firat University, Elazig 23119, Turkey; ksahin@firat.edu.tr (K.S.)

\section{Abstract}

Here, we demonstrate that our anti-sepsis and COVID-19 drug candidate Rejuveinix (RJX) substantially improves the survival outcome in the LPS-GalN animal model of sepsis and multi-organ failure. One hundred (100) percent (\%) of untreated control mice remained alive throughout the experiment. By comparison, $100 \%$ of LPS-GalN injected mice died at a median of 4.6 hours. In contrast to the invariably fatal treatment outcome of vehicle-treated control mice, $40 \%$ of mice treated with $\mathrm{RJX}(\mathrm{n}=25)$ remained alive with a 2.4-fold longer median time survival time of 10.9 hours (Log-rank $X^{2}=20.60, P<0.0001$ ). Notably, RJX increased the tissue levels of antioxidant enzymes SOD, CAT, and GSH-Px, and reduced oxidative stress in the brain. These findings demonstrate the clinical impact potential of RJX as a neuroprotective COVID-19 and sepsis drug candidate.

Keywords: Sepsis, COVID-19, Oxidative stress, Stroke

*Correspondence: fatih.uckun@reven.com 


\section{Introduction}

Severe viral sepsis caused by SARS-CoV-2, the causative agent of coronavirus disease 2019 (COVID-19), shows a rapid progression associated with a cytokine release syndrome (CRS) and a high case fatality rate due to the development of ARDS and multi-organ failure in high-risk COVID-19 patients $(1,2)$. Our anti-sepsis drug candidate, Rejuveinix (RJX), is a rationally-designed formulation of naturally occurring anti-oxidants and anti-inflammatory compounds with a significant clinical impact potential for COVID-19 associated viral sepsis $(3,4)$. RJX showed a very favorable clinical safety profile in a recently completed double-blind, placebo-controlled, randomized, two-part, ascending dose-escalation Phase 1 study in healthy volunteers (Protocol No. RPI003; ClinicalTrials.gov Identifier: NCT03680105) [18]. It has now entered a randomized double-blind, placebo-controlled Phase 2 study in hospitalized patients with critical COVID-19.

More than a third of patients with COVID-19, especially those with severe to critical COVID-19 who managed on an intensive care unit (ICU) develop central nervous system (CNS) symptoms and signs (e.g., headache, dizziness, ataxia, seizure, delirium, confusion, impaired consciousness) consistent with CNS involvement and /or neurological complications (6-13). Cerebrovascular complications of viral sepsis, including ischemic or hemorrhagic stroke, CNS involvement in CRS, hypoxia as well as the interplay of comorbidities have been implicated as contributing factors (6-13). Due to the neuro-invasive capability of SARS-CoV-2, acute disseminated encephalomyelitis (ADEM) and viral encephalitis have also been suspected in some patients (13).

Oxidative stress caused by the massive production of reactive oxygen species (ROS) is thought to play a major role in the pathogenesis of severe viral sepsis in COVID-19 (14). Chaudry et al. recently proposed that reactive oxygen intermediates and oxidative stress may play an important role in the pathophysiology of COVID-19 associated CNS disease, reminiscent of their role in Parkinson's disease (15). Notably, RJX exhibited potent anti-oxidant activity and mitigated lipid peroxidation in both prophylactic and therapeutic settings, as reflected by significantly decreased tissue MDA levels and normalization of the tissue levels of the antioxidant enzymes SOD, CAT, and GSH-Px as well as ascorbic acid (5). The primary objective of the present study was to obtain experimental proof of concept that RJX can mitigate sepsis-associated oxidative stress in the brain. 


\section{Materials and Methods}

\section{Rejuveinix (RJX)}

RJX is a proprietary composition of naturally occurring anti-oxidants and antiinflammatory agents which, in combination, provide potent and immediate tissue protection. Its ingredients include ascorbic acid, magnesium sulfate heptahydrate, cyanocobalamin, thiamine hydrochloride, riboflavin 5' phosphate, niacinamide, pyridoxine hydrochloride, and calcium Dpantothenate. RJX is a two-vial system and $A$ and $B$ are each of the two vials. Vial $A$ contains the active ingredients and minerals whereas Vial $B$ contains the buffer, sodium bicarbonate as the Vial A content is acidic (5).

\section{LPS-GaIN Model of Fatal Cytokine Storm, Sepsis, and Multi-organ Failure}

The ability of RJX, DEX and RJX plus DEX to prevent fatal shock, ARDS, and multiorgan failure was examined in the well-established LPS-GaIN model (5). In this model, LPS is combined with GalN, which further sensitizes mice to LPS-induced systemic inflammatory syndrome and multi-organ failure. The research protocol was approved by the Animal Care and Use Committee of Firat University (Project No. 04052020-391-046). Each mouse received a 500 $\mu \mathrm{L}$ i.p. injection of LPS-GalN (consisting of $100 \mathrm{ng}$ of LPS plus $8 \mathrm{mg}$ of D-galactosamine). Vehicle control mice were treated with $0.5 \mathrm{~mL}$ normal saline (NS) intraperitoneally (i.p) 2 hours before and 2 hours after the i.p injection of LPS-GalN. Test mice received $0.7 \mathrm{~mL} / \mathrm{kg}$ RJX dose ( 2 hours before and 2 hours after LPS-GalN. Mice were monitored for mortality for $24 \mathrm{~h}$. The Kaplan-Meier method, log-rank chi-square test, was used to analyze the $24 \mathrm{~h}$ survival outcomes of mice in the different treatment groups. At the time of death, brains were harvested for biochemical studies.

Lipid peroxidation as a biomarker of oxidative stress was determined and expressed as the amount of malondialdehyde (MDA, nmol/g tissue) in the brain, as previously described (5). The enzymatic activities of SOD, CAT and GSH-Px in the brain specimens were determined using the commercially available kits (Cayman Chemical, Ann Arbor, MI, USA) according to the manufacturer's procedures (5).

\section{Statistical Analyses}

Statistical analyses employed standard methods, including analysis of variance (ANOVA) and/or, nonparametric analysis of variance (Kruskall-Wallis) using the SPSS statistical program (IBM, SPPS Version 21), as reported (5). Furthermore, the Kaplan-Meier 
method, log-rank chi-square test, was used to investigate survival and fatality in each group. Pvalues $<0.05$ were considered significant.

\section{Results}

RJX improves survival outcome after LPS-GalN induced sepsis.

One hundred (100) percent (\%) of untreated control mice remained alive throughout the experiment. By comparison, $100 \%$ of LPS-GalN injected mice died at a median of 4.6 hours (Figure 1). RJX was examined for its protective activity at a dose level, which is $>10$-fold lower than its maximum tolerated dose (MTD) of $0.759 \mathrm{~mL} / \mathrm{kg}$ for human subjects (viz.; $4.2 \mathrm{~mL} / \mathrm{kg}$ of a 6-fold diluted solution). RJX-treated mice had an improved survival outcome after being injected with LPS-GaIN. In contrast to the invariably fatal treatment outcome of vehicle-treated control mice, $40 \%$ of mice treated with $\mathrm{RJX}(\mathrm{n}=25)$ remained alive with a 2.4 -fold longer median time survival time of 10.9 hours (Log-rank $X^{2}=20.60, P<0.0001$ ) (Figure 1).

$R J X$ reduces the oxidative stress in the brain after LPS-GalN induced sepsis.

No histopathologic brain lesions were observed in any of the mice challenged with LPSGalN. However, the brain MDA levels measuring lipid peroxidation were markedly elevated, and the levels of the antioxidant enzymes SOD, CAT, and GSH-Px in the brain were markedly reduced in LPS-GalN treated mice consistent with severe oxidative stress (Figure 2). RJX decreased the brain MDA levels and normalized in a dose-dependent manner the reduced levels of the antioxidant enzymes SOD, CAT, and GSH-Px.

\section{Discussion}

Patients with high-risk COVID-19 are in urgent need of treatment platforms capable of preventing the disease progression and/or reducing the case mortality rate by stopping or reversing the pulmonary as well as systemic inflammatory process that cause the ARDS and culminate in multi-organ failure $(1,2,16)$. Here we extend our previous study and provide experimental evidence that RJX is capable of improving the survival outcome in the LPS-GalN mouse model of fatal sepsis.

SOD, CAT, and GSH-Px are three pivotal antioxidant defense enzymes, and their levels are altered by the level of oxidative stress that is a hallmark of severe inflammation of sepsis (14). Oxidative stress caused by massive production of reactive oxygen species (ROS) is 
thought to play a major role in the pathogenesis of severe viral sepsis in COVID-19 as well (14). The MDA levels were markedly elevated in the brain specimens of the LPS-GalN challenged mice which is consistent with increased lipid peroxidation. In parallel, the levels of the antioxidant enzymes SOD, CAT, and GSH-Px were profoundly suppressed due to severe oxidative stress. RJX exhibited potent anti-oxidant activity and mitigated lipid peroxidation, as reflected by significantly decreased tissue MDA levels and normalization of the tissue levels of the antioxidant enzymes SOD, CAT, and GSH-Px as well as ascorbic acid. We hypothesize that RJX will shorten the time to resolution of ARDS and viral sepsis in COVID-19 patients by preventing the development of a fulminant cytokine storm as well as reversing the cytokinemediated multi-system inflammatory process and oxidative stress, thereby mitigating the inflammatory organ injury.

Oxidative stress owing to mitochondrial dysfunction has been implicated in the pathophysiology of Alzheimer's disease (AD), Parkinson's disease (PD), and Huntington's disease (HD). Notably, in a mouse CNS model of severe oxidative stress, RJX rapidly and substantially increased the levels of the anti-oxidant enzymes SOD, CAT, GSH-Px that were reduced in the brains of LPS-GaIN treated mice consistent with severe oxidative stress. Our results indicate that RJX could have therapeutic utility in the treatment of $A D, P D, H D$, and amyotrophic lateral sclerosis (ALS). Furthermore, because of the well-established role of oxidative stress in the development and progression of ischemic stroke, RJX could significantly improve the standard of care for stroke as well.

Author contributions: Each author has made significant and substantive contributions to the study, reviewed and revised the manuscript, provided final approval for submission of the final version. No medical writer was involved. F.M.U conceived the study, designed the evaluations reported in this paper, directed the data compilation and analysis, analyzed the data, and prepared the initial draft of the manuscript. Each author had access to the source data used in the analyses.

Funding/Support: This study was funded by Reven Pharmaceuticals, LLC, a wholly-owned subsidiary of Reven Holdings Inc.

Conflicts of Interest: F.M.U. and M.V are employees of Reven Pharmaceuticals, the sponsor for the clinical development of RJX. M.T., M.G, and K.S declare no current competing financial interests. 
Role of the Funder/Sponsor: The sponsor did not participate in the collection of data. Two of the authors (F.M.U, and M.V) who participated in the analysis and decision to submit the manuscript for publication are affiliated with the sponsor.

\section{References}

1.Uckun FM. Reducing the Fatality Rate of COVID-19 by Applying Clinical Insights From Immuno-Oncology and Lung Transplantation. Front. Pharmacol. 2020; 11:796. doi: 10.3389/fphar.2020.00796

2. Fang J, Liehua D, Liangqing Z, Yin C, Wai CC, Zhengyuan X. Review of the Clinical Characteristics of Coronavirus Disease 2019 (COVID-19) Journal of General Internal Medicine. 2020;35(5):1545-1549. doi: 10.1007/s11606-020-05762-w.

3. Cabala K, Earabino J, Pacult P, Uckun FM. Rationale For A Randomized, PlaceboControlled, Phase 2 Study Of Rejuveinix (RJX) in COVID-19 Patients With Acute Lung Injury And Hypoxemic Respiratory Failure. Clinical Investigation (London) 2020; 10(2): 185-189

4. Uckun FM, Ervin J, Sahin K, Powell J, Pizzimenti N, Earabino J, Van Wyk H, Van Wyk M, Pacult P, Sahin TK, Cabala K. Clinical Impact Potential Of Rejuveinix (RJX) For Prevention Of Fatal Acute Respiratory Distress Syndrome (ARDS) And Multi-Organ Failure In Covid-19 Patients. Clinical Investigation (London) 2020; 10(2): 177-184

5. Uckun FM, Carlson J, Orhan C, Powell J, Pizzimenti NM, Van Wyk H, Ozercan IH, Volk M, Sahin K. Rejuveinix Shows a Favorable Clinical Safety Profile in Human Subjects and Exhibits Potent Preclinical Protective Activity in the Lipopolysaccharide-Galactosamine Mouse Model of Acute Respiratory Distress Syndrome and Multi-Organ Failure. Front. Pharmacol. 2020; 11:594321. doi: 10.3389/fphar.2020.594321

6. Azhideh A. COVID-19 Neurological Manifestations. Int Clin Neurosci J. 2020;7(2):54. doi: 10.34172/icnj.2020.01.

7. Zangbar HS, Ali Gorji A, Ghadiri T. A Review on the Neurological Manifestations of COVID19 Infection: a Mechanistic View. Mol Neurobiol. 2020 Sep 26 : 1-14. doi: 10.1007/s12035-02002149-0 [Epub ahead of print]

8. Wu Y, Xu X, Chen Z, Duan J, Hashimoto K, Yang L et al (2020) Nervous system involvement after infection with COVID-19 and other coronaviruses. Brain Behav Immun.

10.1016/j.bbi.2020.03.031 
9. Achar A, Ghosh C. COVID-19-Associated Neurological Disorders: The Potential Route of CNS Invasion and Blood-Brain Relevance. Cells. 2020 Oct 27;9(11):2360. doi:

$10.3390 /$ cells 9112360 .

10. Verma K, Prasad DN, Kumar B, Kohli E. Brain and COVID-19 Crosstalk: Pathophysiological and Psychological Manifestations. ACS Chem Neurosci. 2020 Oct 21;11(20):3194-3203. doi: 10.1021/acschemneuro.0c00446. Epub 2020 Oct 2.

11. Alharthy A, Faqihi F, Memish ZA, Karakitsos D. Fragile Endothelium and Brain Dysregulated Neurochemical Activity in COVID-19. ACS Chem Neurosci. 2020 Aug 5;11(15):2159-2162. doi: 10.1021/acschemneuro.0c00437. Epub 2020 Jul 27.

12. Niazkar HR, Zibaee B, Nasimi A, Bahri N. The neurological manifestations of COVID-19: a review article. Neurol Sci. 2020 Jul;41(7):1667-1671. doi: 10.1007/s10072-020-04486-3. Epub 2020 Jun 1. PMID: 32483687; PMCID: PMC7262683.

13. Hassanzadeh K, Perez Pena H, Dragotto J, Buccarello L, lorio F, Pieraccini S, Sancini G, Feligioni M. Considerations around the SARS-CoV-2 Spike Protein with Particular Attention to COVID-19 Brain Infection and Neurological Symptoms. ACS Chem Neurosci. 2020 Aug 5;11(15):2361-2369. doi: 10.1021/acschemneuro.0c00373. Epub 2020 Jul 21.

14. Schönrich G, Raftery MJ, Samstag Y. Devilishly radical NETwork in COVID-19: Oxidative stress, neutrophil extracellular traps (NETs), and T cell suppression. Adv Biol Regul. 2020;77:100741.

15. Chaudhary Z, Klenja D, Janjua N, Cami-Kobeci G, Ahmed BY. COVID-19 and Parkinson's Disease: Shared Inflammatory Pathways Under Oxidative Stress. Brain Sci. 2020; 10(11):807. doi: 10.3390/brainsci10110807.

16. Zumla A, Hui DS., Azhar El, Memish ZA, Mauerer M. Reducing mortality from 2019-nCoV: host-directed therapies should be an option. Lancet. 2020;395(10224):e35-e36. doi: 10.1016/S0140-6736(20)30305-6. 


\section{Figures}

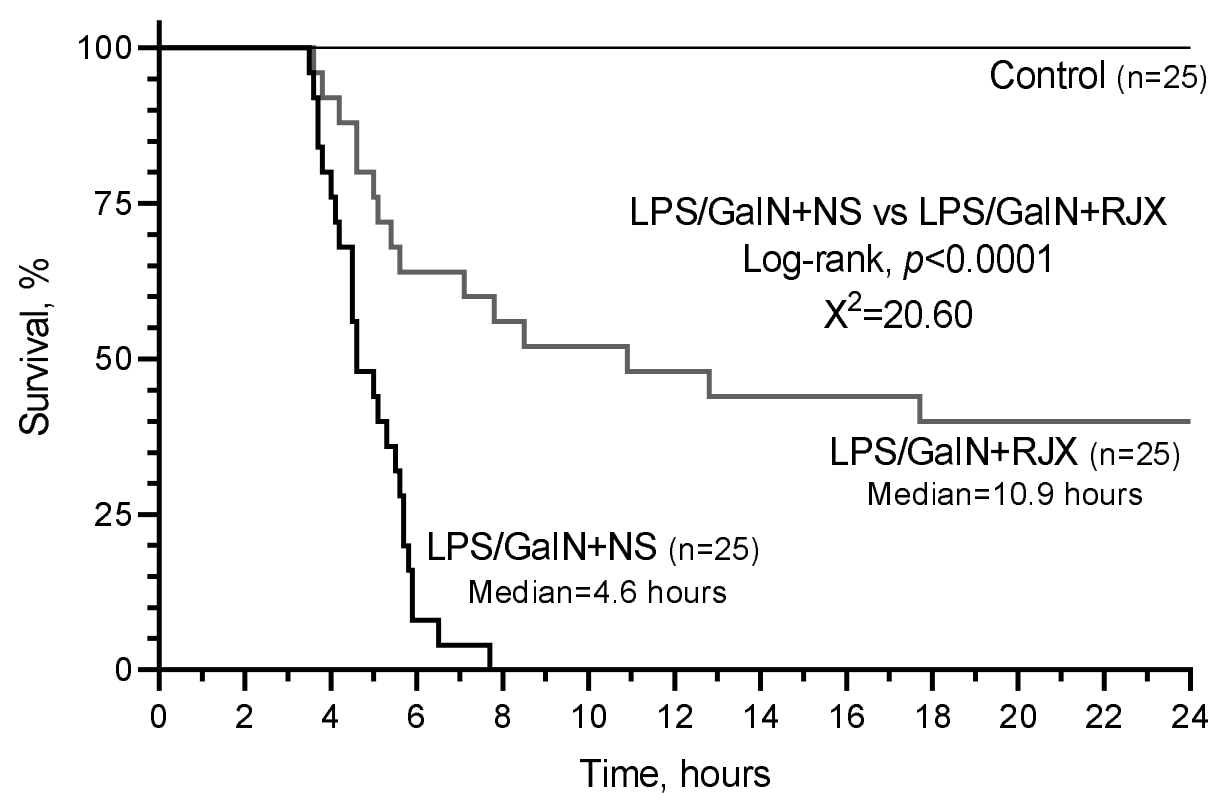

Figure 1. In Vivo Protective Activity of RJX in the LPS-GALN challenged mice. Groups of $25 \mathrm{BALB} / \mathrm{C}$ mice were treated with i.p injections of 6-fold diluted RJX (4.2 mL/kg, $0.5 \mathrm{ml} / \mathrm{mouse}$ ) or vehicle (NS) 2 hours before or post-injection of LPS-GaIN. Except for untreated mice (Control), each mouse received $0.5 \mathrm{ml}$ of LPS-GalN (consisting of $100 \mathrm{ng}$ of LPS plus $8 \mathrm{mg}$ of D-galactosamine) i.p. Survival is shown as a function of time after the LPS-GalN challenge. Depicted are the survival curves for each group along with the median survival times and the log-rank P-value for the comparison of the LPS-GalN+RJX group versus the LPS/GaIN+NS group. 

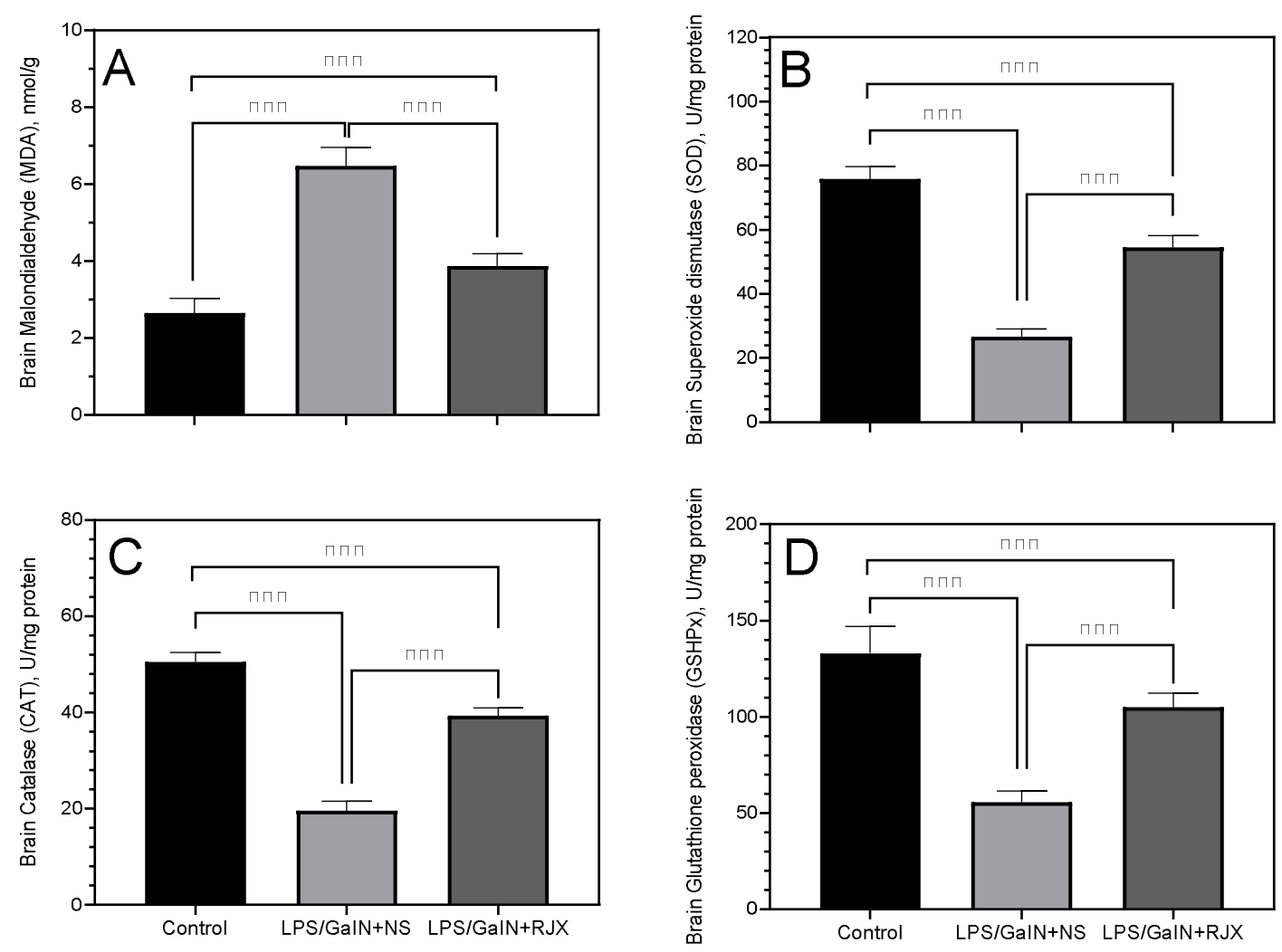

Figure 2. Effect of Rejuveinix (RJX) on brain malondialdehyde (MDA; Panel A), superoxide dismutase (SOD; Panel B), catalase (CAT; Panel C) and Glutathione peroxidase (GSHPx; Panel D) in the lipopolysaccharide-galactosamine (LPS-GaIN) challenged mice. Each bar represents the mean and standard deviation for the measured parameter of mice from each specific treatment group (6 mice/group). BALB/C mice were treated with i.p injections of 6-fold diluted RJX $(4.2 \mathrm{~mL} / \mathrm{kg}, 0.5 \mathrm{ml} / \mathrm{mouse})$ or vehicle (NS) 2 hours before or post-injection of LPS-GalN. Except for untreated mice (Control), each mouse received $0.5 \mathrm{ml}$ of LPS-GalN (consisting of $100 \mathrm{ng}$ of LPS plus $8 \mathrm{mg}$ of D-galactosamine) i.p. (ANOVA and Tukey's post-hoc test. Statistical significance between groups is shown by: ${ }^{\star \star \star} \mathrm{P}<$ 0.001). 